Int. J. Dev. Biol. 54: 1473-1480 (2010)

doi: $10.1387 /$ ijdb. 103071 as

\title{
Glucocorticoid receptor antagonizes EGFR function to regulate eyelid development
}

\author{
ANA SANCHIS\#, PILAR BAYO\#, LISA M. SEVILLA and PALOMA PÉREZ* \\ Instituto de Biomedicina de Valencia, Consejo Superior de Investigaciones Científicas (IBV-CSIC), \\ Valencia, Spain
}

\begin{abstract}
The glucocorticoid receptor (GR) plays a crucial role in epidermal morphogenesis during embryonic development, as demonstrated by analyzing genetically modified mouse models of GR gain- and loss-of-function. Eyelid formation constitutes a useful model to study epithelial development, as it requires coordinated regulation of keratinocyte proliferation, apoptosis and migration. We have analyzed this biological process in $\mathbf{G R}^{-/-}$embryos during ontogeny. Our data demonstrate that GR deficiency results in delayed and impaired eyelid closure, as illustrated by increased keratinocyte proliferation and apoptosis along with impaired differentiation in $\mathrm{GR}^{-/-}$eyelid epithelial cells. These defects are due, at least in part, to the lack of antagonism between GR and epidermal growth factor receptor (EGFR) signaling, causing sustained activation of the MAPK/AP-1 pathway and the upregulation of keratin K6 at embryonic stage E18.5. Additionally, we demonstrate that GR regulates epithelial cell migration in vitro by interfering with EGFR-mediated signaling. Overall, GR/EGFR antagonism appears as a major mechanism regulating ocular epithelial development.
\end{abstract}

KEY WORDS: glucocorticoid receptor, EGFR, genetically modified mice, epithelial cell, eye development

\section{Introduction}

It is known that the glucocorticoid receptor (GR) plays a crucial role during embryogenesis, since this ligand-activated transcription factor is required for maturation of vital organs such as the lung, heart, kidney, gut and epidermis (reviewed in Wintermantel et al., 2004; Revollo and Cidlowski, 2009). GR exerts its biological effects through two different mechanisms that involve DNA binding-dependent and -independent actions, which can be genetically separated and are commonly refered to as transactivation and transrepression functions. In fact, knock-out $\mathrm{GR}^{-/-}$mice die perinatally whereas mice carrying a point mutation which abrogates the dimerization- dependent DNA binding of GR (GR $\left.{ }^{\mathrm{dim} / \mathrm{dim}}\right)$ are viable (Cole et al., 1995; Reichardt et al., 1998). This distinction, however, is not so clear-cut since GR monomers are also able to bind certain gene promoters and thus, regulate gene transcription (Adams et al., 2003; Rogatsky et al., 2003): In the last years, we have demonstrated that GR is a key player in epithelial development by analyzing genetically modified mouse models of GR gain- and loss-of-function (Pérez et al., 2001; Cascallana et al., 2003; Cascallana et al., 2005; Donet et al.,
2008; Bayo et al., 2008). We have recently reported that only the epidermis of $\mathrm{GR}^{-/-}$but not $\mathrm{GR}^{\mathrm{dim} / \mathrm{dim}}$ embryos shows major defects, suggesting that dimerization dependent DNA binding by GR is dispensible for epidermal development (Bayo et al., 2008). In addition, we have shown that transgenic mice with keratinocytetargeted overexpression of GR (K5-GR mice) featured numerous epithelial defects including epidermal defects and an eyelid opening at birth phenotype (EOB). Overexpression of GR transrepression function in keratinocytes (K5-GR-TR mice) also elicited epithelial alterations that partially overlapped with those found in K5-GR mice (Cascallana et al., 2005; Donet et al., 2008). Remarkably, K5-GR-TR mice featured an EOB phenotype identical to K5-GR mice, indicating that the transrepression function of the GR is sufficient to cause these epithelial ocular anomalies (Donet et al., 2008).

Eyelid formation represents a useful model to study epithelial development since this biological process requires spatio-tempoAbbreviations used in this paper: EGFR, epidermal growth factor receptor; GC,
glucocorticoid hormone; GR, glucocorticoid receptor.

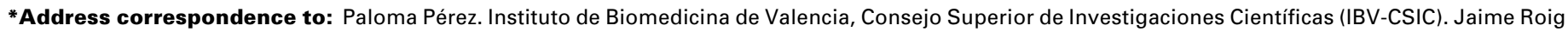
11, E-46010 Valencia, Spain. Fax: +34-96-369-0800.e-mail: pperez@ibv.csic.es

\#Note: Both authors contributed equally to this paper. 
ral coordination of cellular proliferation, migration, differentiation and apoptosis. In the mouse embryo, eyelid formation starts at midgestation, when small invaginations of the surface ectoderm begin to grow (E12.5), become distinguishable as protruding edges (E14.5-E15.5) and gradually cover the corneal surface until they fuse at E16.5. At E18.5, eyelids are unequivocally closed and start to separate postnatally (P3-P5) until they reopen around P12 (Kaufman and Bard, 1999). In this work, we analyzed the consequences of GR functional inactivation in ocular development by using $\mathrm{GR}^{-/-}$mice.'Our data show that GR is required to modulate eyelid epithelial morphogenesis through regulation of keratinocyte proliferation, apoptosis, differentiation and migration. It is known that GR suppresses keratin expression of either mitotically-active basal keratinocytes (K5 and K14) or migrationassociated keratins $\mathrm{K} 6$ and $\mathrm{K} 16$. The mechanisms involve binding of GR to AP-1 and interaction of four GR monomers with k6 promoter, respectively (Radoja et al., 2000; De Bosscher and Haegeman, 2009). Accordingly, K5 and K6 expression was altered in $\mathrm{GR}^{-/-}$epithelia. These actions were mediated, at least in part, through interference with EGFR signaling, the loss of which results in increased EGFR and MAPK/AP-1 activation and sustained expression of the keratin $\mathrm{K} 6$. In vitro wound healing assays demonstrated that constitutive GR overexpression in keratinocytes drastically impairs cell migration and interferes with EGFR function suggesting that lack of cross-talk between GR and EGFR pathway is one of the mechanisms causing delayed eyelid closure in $\mathrm{GR}^{-/-}$embryos.

\section{Results}

We have anayzed eyelid closure in $\mathrm{GR}^{-/-}$embryos as in vivo model to address the impact of GR loss-of-function in epithelial morphogenesis. Macroscopically, $\mathrm{GR}^{-/-}$embryos showed open eyelids at embryonic days E16.5 and E18.5. A detailed histopathological analysis of wt and $\mathrm{GR}^{-/-}$littermates at distinct timepoints showed severe anomalies in the eyelid formation of GR-deficient mice throughout development (Fig. 1A, compare a$d$ and $\left.a^{\prime}-d^{\prime}\right)$. In a wt developing mouse, eyelid closure is normally completed in 24h, coinciding with the E15.5-E16.5 transition (Fig. $1 \mathrm{~A}$; $\mathrm{a}, \mathrm{b}-\mathrm{d}$; asterisks indicate fused eyelids in $\mathrm{GR}^{+/+}$). In contrast,

A
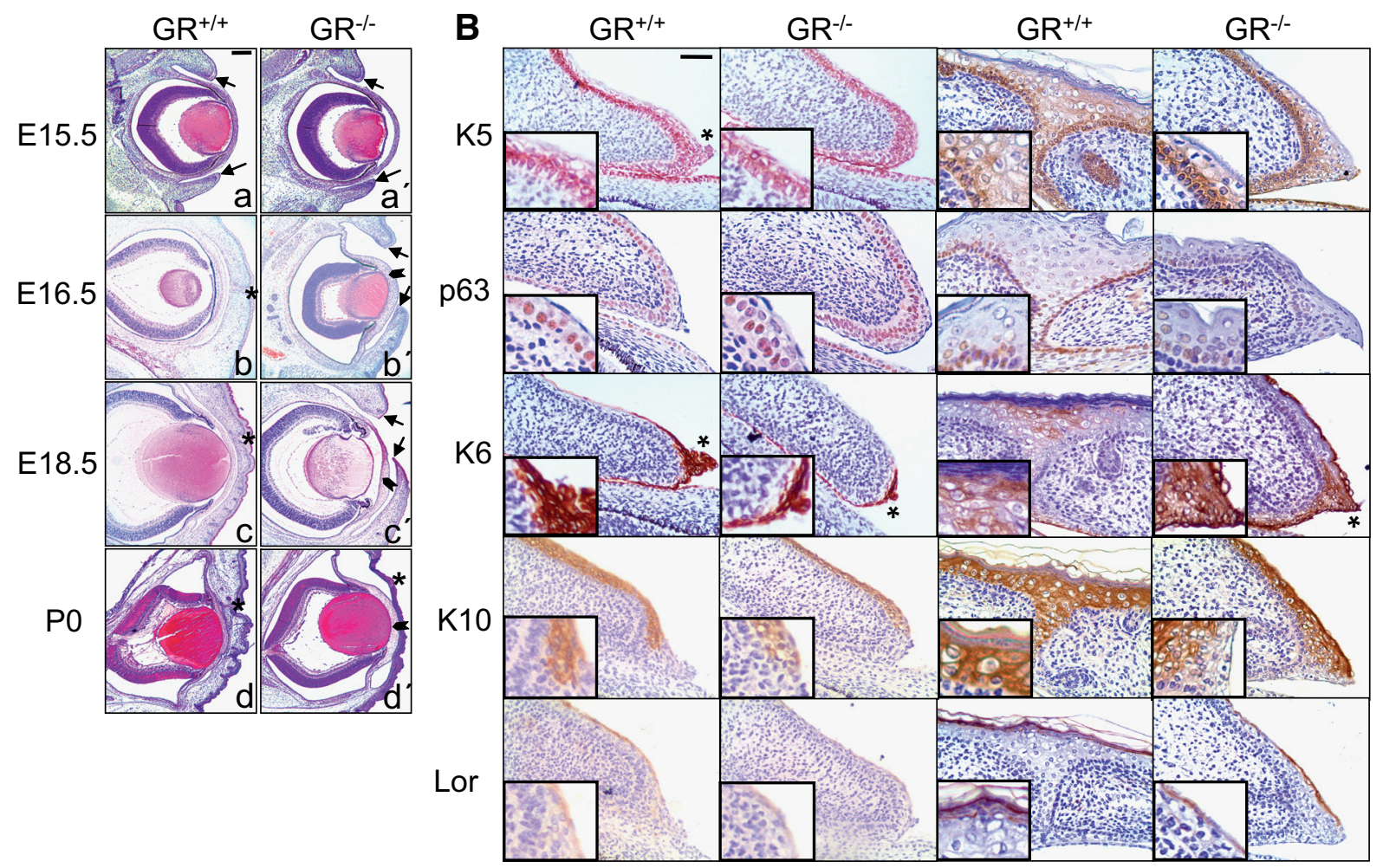

E15.5

E18.5

Fig. 1. Delayed and impaired eyelid closure in glucocorticoid receptor (GR) null mice. (A) Histopatholgical analysis of wt and $G R^{-1-}$ embryos at distinct developmental timepoints. Eyelid closure is normally completed at E15.5-E16.5 transition in wt embryos and eyelids remain fused until postnatal age (a-d). In contrast, GR ${ }^{-1}$ embryos exhibited unfused eyelids at E16.5 and E18.5 (a'-c', arrows) along with an abnormal corneal stroma with increased cellularity (b', $c^{\prime}$, arrowheads). GR ${ }^{-/}$newborn mice showed closed eyelids with abnormal epithelia and atypical corneal stroma (d', asterisk and arrowhead, respectively). Bar: $200 \mu \mathrm{m}$. (B) Abnormal morphogenesis of eyelid epithelial cells in GR- early (E15.5) and late (E18.5) embryos. Immunostaining for K5, K6, p63, K10 and loricrin was performed using specific antibodies. Note that both $K 5$ and p63 expression stained two-to three suprabasal layers of eyelid epithelia in $G R^{-1}$ embryos as compared to more restricted labeling at the fused eyelids of GR ${ }^{+/+}$individuals (K5 and p63; see inset). Increased K6 expression at the eyelids tips of E15.5 (asterisks) was observed in wt mice. Elevated levels of K6 were detected in the unfused $G R^{--}$eyelids at E18.5, as compared with restricted K6 expression at closed eyelids of GR+/+ mice. Abnormal K10 and loricrin staining was apparent in $\mathrm{GR}^{-/}$eyelid epidermis relative to $\mathrm{G} R^{+/+}$mice (K10 and loricrin; see inset). Bar: $100 \mu \mathrm{m}$. 


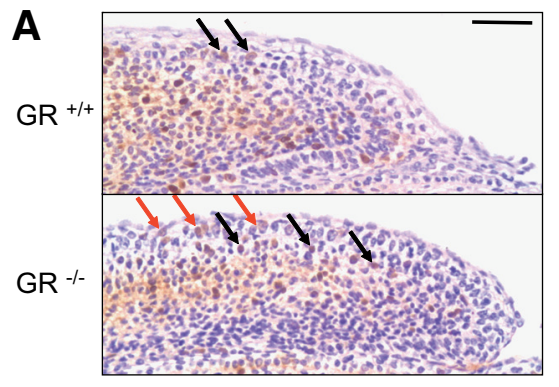

B
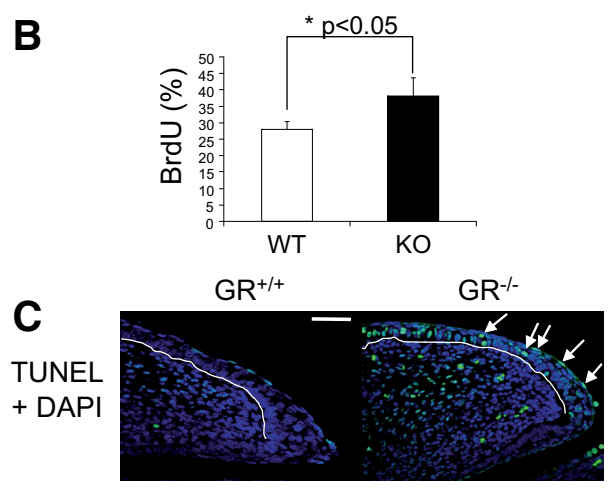

D
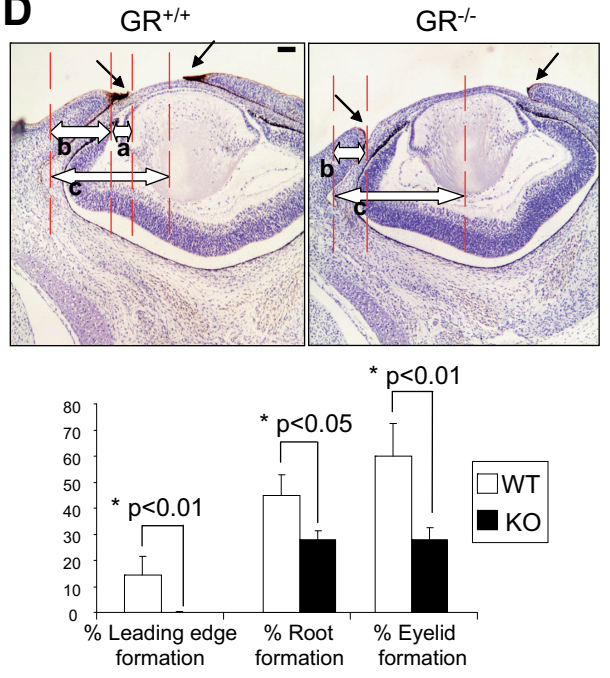

$[\mathrm{a} / \mathrm{c}] \times 100=$ Leading edge formation $(\%)$ [b/c] $\times 100=$ Root formation (\%) $[(a+b) / c] \times 100=$ Eyelid formation $(\%)$

Fig. 2. Increased proliferation and apoptosis in $\mathrm{GR}^{-/-}$eyelid epithelial cells and impaired eyelid formation in $\mathbf{G R}^{-/-}$mice. (A) In vivo BrdU labeling showed increased epithelial proliferation in $\mathrm{GR}^{-/}$relative to $\mathrm{GR}^{+/+} \mathrm{E} 15.5 \mathrm{em}$ bryos. Note that augmented keratinocyte proliferation was detected in eyelid basal (black arrows) and suprabasal (red arrows) cells. (B) Quantitative differences were statistically significant, $p<0.05$. (C) TUNEL staining shows augmented apoptosis in all eyelid epithelial layers of $\mathrm{GR}^{-/}$relative to $\mathrm{GR}^{+/+}$E15.5 embryos. Arrows point to TUNEL-positive cells and the line denotes the basal membrane of the eyelid epithelium. (D) Quantitation of formation of eyelid, leading edge and root in $G R^{-1}$ vs wt E15.5 littermates (five of each genotype). Measurements were done as summarized in Materials and Methods. Briefly, the migrating epithelial cells were identified by $K 6$ immunostaining. Parametera is the distance of migration; parameter $\mathrm{b}$ the distance between the axis delimitating the conjunctival sac and the first $K 6$-stained cells, and parameter $\mathrm{c}$ is the distance between the axis delimitating the conjunctival sac and the center of the cornea. The Student t test was used to calculate statistical significance $(n=5$, each genotype) and differences considered to be statistically significant when p<0.05. Bar: $100 \mu \mathrm{m}$.

$\mathrm{GR}^{-/-}$embryos revealed unfused eyelids at E16.5 and even at E18.5 (Fig. 1A; b', c', arrows) along with an abnormal corneal stroma with increased cellularity (Fig. 1A; $b^{\prime}, c^{\prime}$, arrowheads). The eyelids of $\mathrm{GR}^{-/}$newborn mice were closed but the epithelia appeared abnormally enlarged and undifferentiated (Fig. 1A; compare $d$ and $d^{\prime}$ ) and an atypical corneal stroma persisted. Penetrance of the reported ocular phenotype in $\mathrm{GR}^{-1-}$ mice was $89 \%(n=43)$, with either one or two eyes affected $(54.55 \%$ and $45.45 \%$, respectively).

As an attempt to understand how loss of GR is causing these defects, we analyzed the expression of markers of keratinocyte proliferation, migration and differentiation in the eyelid epithelia of early (E15.5) and late (E18.5) embryos by immunostaining (Fig. 1B). In wt embryos, $\mathrm{K} 5$ expression was apparent in all epithelia basal cells of the eyelids, cornea and conjunctiva (Fig. 1B, K5). In $\mathrm{GR}^{-/-}$littermates, labeling of these epithelial cell layers was similar, although additional K5 expression was detected suprabasally in the eyelid epithelia (Fig. 1B, K5, see inset). The epithelial-specific marker p63 was detected in keratinocytes of $\mathrm{GR}^{+/+}$and $\mathrm{GR}^{-/-}$embryo eyelids and cornea. However, p63 immunostaining at the leading edge of early and late $\mathrm{GR}^{-/}$embryo eyelids was more suprabasal as compared to the more restricted p63 labeling at wt eyelid tips (Fig. 1B, p63).

In E15.5 wt mice, K6 was strongly expressed at the eyelids tips, defining the migrating cells at the leading edge of this epithelium (Fig. 1B, K6, asterisk). The conjuctival epithelium was also K6positive (not shown). Notably, at E18.5, where cell migration is no longer required since eyelids are already fused, $\mathrm{K} 6 \mathrm{immunostaining}$ was restricted to the closed eyelids (Fig. 1B). In contrast, $\mathrm{GR}^{-1-}$ embryos stained weakly for $\mathrm{K} 6$ at the eyelid borders at E15.5 whereas an abnormally strong $\mathrm{K} 6$ signal was detected at $\mathrm{E} 18.5$, both at the tip of unfused $\mathrm{GR}^{-/}$eyelids and in conjuctival epithelium (Fig. 1B, K6, asterisk and data not shown). The observed K6 expression pattern indicates a delay in the ocular epithelia development of $\mathrm{GR}^{-/-}$mice.
Previous reports described that eyelid fusion precedes epithelial differentiation, since positive K10 staining was detected in epithelial cells of wt embryos only after eyelid closure (Zhang et al., 2005). We examined K10 and loricrin expression and found that in E15.5 wt embryos, both markers were present in suprabasal eyelid epithelia although absent at the leading edge. In E18.5 wt embryos, only suprabasal cells of the fused eyelids stained positive for both markers. In contrast, reduced staining of $\mathrm{K} 10$ and loricrin was apparent in $\mathrm{GR}^{-/-}$eyelid epidermis of early embryos whereas abnormal expression of these proteins persisted in the unfused eyelids from GR ${ }^{-/-}$E18.5 embryos (Fig. 1B, K10 and loricrin).

We further assessed altered proliferation in $\mathrm{GR}^{-/-}$eyelid epithelial cells by measuring in vivo BrdU incorporation in $\mathrm{GR}^{-/-}$vs wt E15.5 embryos, and found qualitative and quantitative differences among them (Fig. 2A, B). In wt embryos, most BrdUpositive keratinocytes were detected in the basal cell of the eyelid epithelia (black arrows). In contrast, augmented keratinocyte proliferation was detected in basal (black arrows) and suprabasal (red arrows) cells of $\mathrm{GR}^{-/}$eyelids. When comparing the proliferation of eyelid basal cells, differences were found statistically significant (Fig. 2A, 38.1\% vs $28 \%$, respectively, $\mathrm{p}<0.05)$. At later developmental stages (E18.5), we could not detect increased proliferation of the eyelid basal keratinocytes of $\mathrm{GR}^{-1-}$ relative to wt (data not shown). However, we found BrdU-positive nuclei in the suprabasal layers of E18.5GR ${ }^{-/-}$eyelids, as occurred in E15.5 GRdeficient embryos. Additionally, we detected augmented apoptosis in all eyelid epithelial layers of GR-deficient embryos by TUNEL staining, in contrast with scarce apoptotic cells in wt littermates (Fig. 2B, arrows). Overall, our results demonstrate that $\mathrm{GR}$ is required for proper proliferation, apoptosis, migration and differentiation of the eyelid epithelial cells of $\mathrm{GR}^{-1-}$ embryos.

To further characterize the alterations in $\mathrm{GR}^{-/-}$eyelid closure, we quantitated several parameters to estimate the percentage of formation of the eyelid, leading edge and root, following the 
methodology described by Mine and co-workers (Mine et al., 2005). For these measurements, we performed K6 immunostaining to compare eyelid closure in $\mathrm{GR}^{-/-}$and wt E15.5 littermates, using K6-positive cells to define the migrating epithelial cells (Fig. 2C). In wt embryos, extension of both leading edge and the root was already evident at E15.5 with estimated percentage of formation of $14.5 \%$ and $45 \%$, respectively (Fig. $2 \mathrm{C}$ ). In sharp contrast, the leading edge of GR null mice was not formed at this stage and it was not apparent until E18.5 (Fig. 1B and data not shown). In addition, root formation was reduced almost two-fold, as compared to wt embryos. These determinations allowed us to quantitate the overall completion in $\mathrm{GR}^{-/}$eyelid formation at E15.5 as of $28 \%$ relative to $60 \%$ in wt (Fig. $2 \mathrm{C}$ ).

It is well known that the epidermal growth factor (EGF) and its receptor (EGFR) play a crucial role in epithelial development (Zenz et al., 2003; Xia and Karin, 2004). The antagonism between GR and EGFR signaling has been demonstrated in different pathophysiological processes. However, this cross-talk has not been examined in eyelid formation. In wt embryos, EGFR was detected at the tip of eyelid epithelial cells around E15.5 but decreased at E18.5, when eyelid closure had completed. In contrast, EGFR protein expression was evident at E15.5 in $\mathrm{GR}^{-1}$ - embryos and remained abnormally high at E18.5, as compared to wt (Fig. 3A, EGFR). The observed elevation in total EGFR levels correlated with increased phosphorylated ( $p-) E G F R$ immunostaining in $\mathrm{GR}^{-/-}$open eyelids at E18.5 (Fig. 3A, p-EGFR). To ascertain whether GR could also regulate EGFR at the transcriptional level, we examined the skin of E18.5 $\mathrm{GR}^{-/-} \mathrm{em}$ bryos as compared to wt littermates by quantitative RT-PCR (Fig.
3B). Our data demonstrated increased egfr mRNA levels in GR/- embryos, thus indicating additional mechanisms of GR/EGFR biological antagonism.

We next examined whether increased EGFR signaling would cause augmented ERK phosphorylation in the developing eyelids of $\mathrm{GR}^{-/}$embryos (Fig. 3C, p-ERK). Despite unchanged total ERK levels in wt and $\mathrm{GR}^{-/-}$embryos (not shown), we detected p-ERK at E15.5 only in GR-deficient eyelids. In E18.5 wt embryos, p-ERK was restricted to the granular layer of closed eyelids whereas high levels were still detected at the eyelid tip of $\mathrm{GR}^{-/-}$embryos. Overall, our results suggest that proper (and transient) regulation of EGFR signaling and its downstream effectors, such as ERK and K6, are required during ocular epithelia development. Abnormally constitutive activation and/or expression of these proteins, as in GRdeficient embryos, likely results in impaired eyelid closure.

The formation of the mouse eyelid during the embryogenesis is similar to the wound healing process since it requires coordinated proliferation, migration and differentiation of keratinocytes (reviewed in Martin and Parkhurst, 2004). We thus examined the role of GR and its interference with EGFR function in epithelial cell migration by in vitro wound healing assays using primary culture keratinocytes (MPKs). Since cell confluence is necessary for these experiments, and given that $\mathrm{GR}^{-/-}$MPKs exhibit abnormal cell growth and apoptosis (Bayo et al., 2008), we evaluated MPKs isolated from K5-GR transgenic mice, in which GR is constitutively active (Pérez et al., 2001). This system allows us to evaluate the role of GR in keratinocyte migration without adding exogenous ligands, which can elicit different actions depending on dosing and kinetics. Fig. 4 summarizes three independent wound scratch
E15.5

A

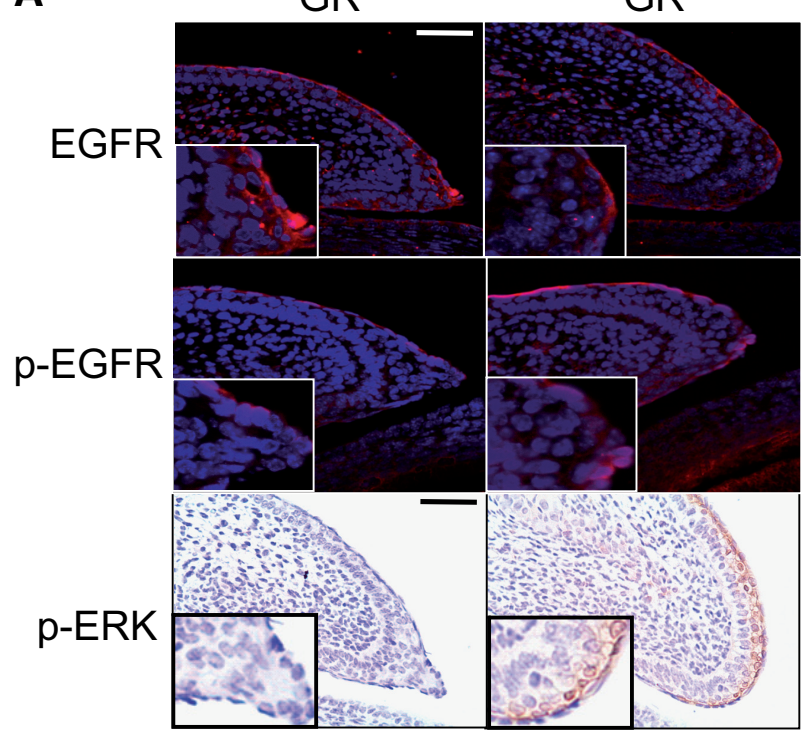

E18.5

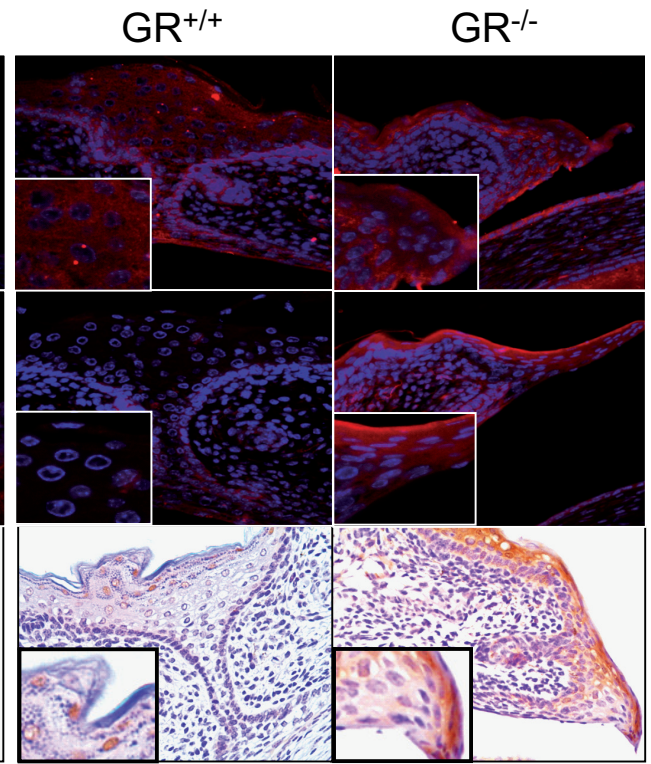

B

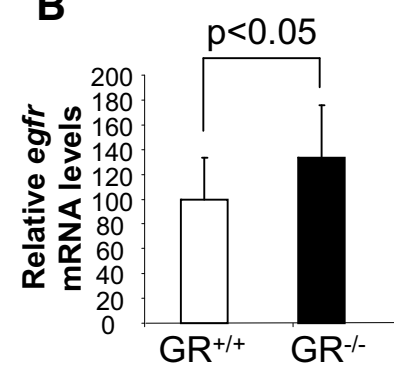

Fig. 3. Glucocorticoid receptor (GR) interferes with EGFR-mediated signaling during eyelid development. (A) Immunofluorescences were performed in $\mathrm{GR}^{-/}$and $G R^{+/+}$embryos at E15.5 and E18.5 with specific antibodies against total EGFR and phosphorylated EGFR (p-EGFR). EGFR expression was slightly induced in $G R^{-/}$relative to $G R^{+/+} E 18.5$ embryos. Moreover, increased $p$-EGFR immunostaining was also detected in GR ${ }^{-1}$ E15.5 and E18.5 eyes. (B) Transcript levels of egfr were examined in skin of $G R^{-/-}$and $G R^{+/+} E 18.5$ embryos by quantitative RT-PCR. Asterisk denote that differences in four individuals of each genotype were statistically significant; (student's t test, $p<0.05$ ). (C) Immunostaining in $G R^{-1}$ and $G R^{+/+}$embryos at E15.5 and E18.5 for phosphorylated (p)-ERK. p-ERK was detected at E15.5 only in GR-deficient eyelids and the phosphorylation was sustained until E18.5, in contrast to wt embryos, where p-ERK was restricted to the granular layer of closed eyelids. Bar: $100 \mu \mathrm{m}$. 


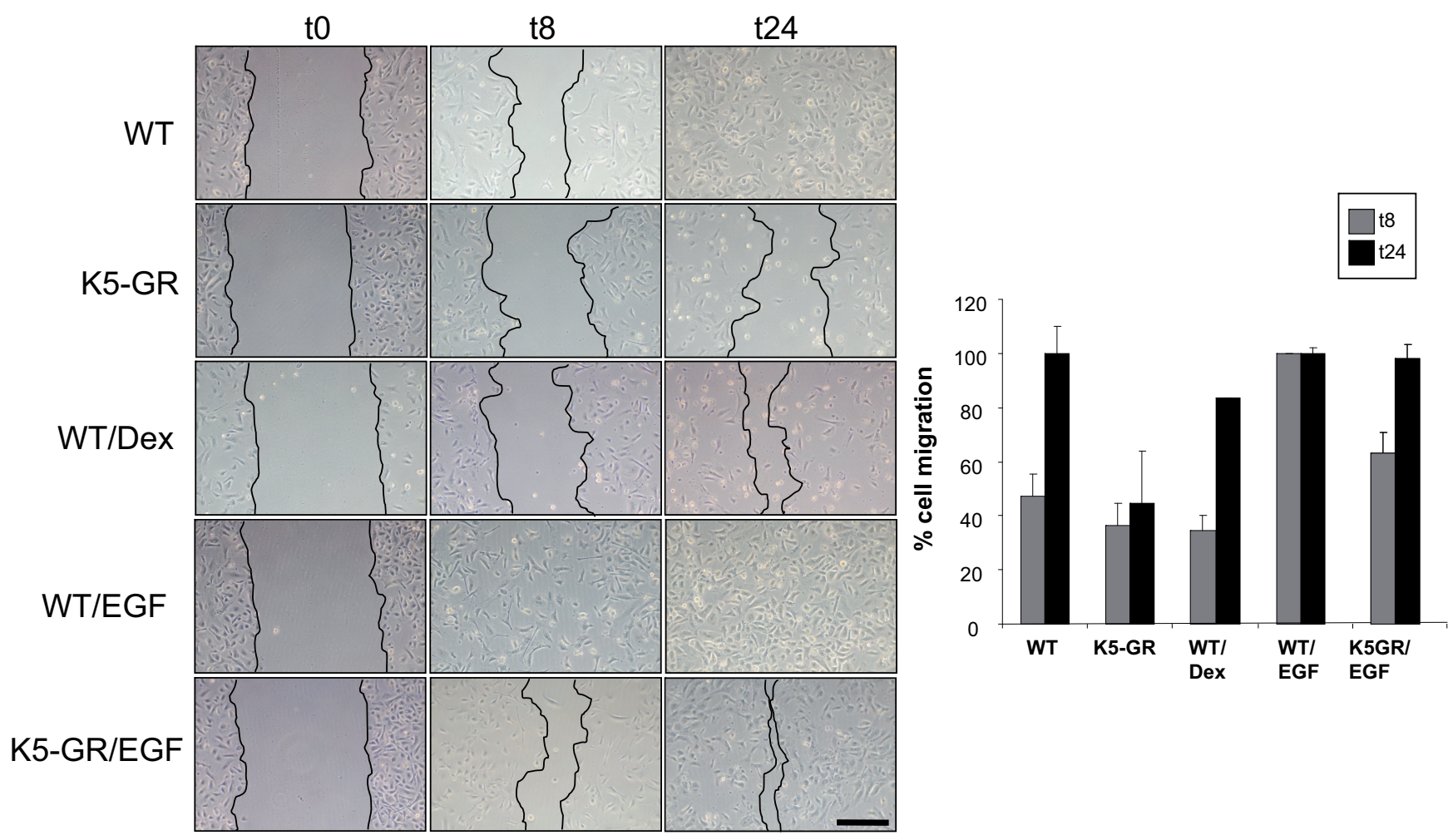

Fig. 4. Glucocorticoid receptor (GR) delays keratinocyte migration in in vitro wound scratch assays. Wound scratch experiments were performed by using MPKs from wt and K5-GR mice and wound closure was evaluated at $8 \mathrm{~h}$ (t8) and 24h (t24). Cells were treated with vehicle, Dex $(100 \mathrm{nM})$ or EGF $(25 \mathrm{ng} / \mathrm{ml})$, as indicated. Note that the delayed keratinocyte migration in K5-GR MPKs was similar to that elicited by Dex treatment of wt cells. EGF accelerates keratinocyte migration in both genotypes although to a lesser extent in K5-GR cells. Microphotographs are representative of three independent experiments. The graph shows the average of three independent experiments. Quantitation of cell migration percentage was estimated as indicated in Materials and Methods. Bar: $100 \mu \mathrm{m}$.

experiments that elicited consistent results. In wt cells, keratinocyte migration was $47.3 \%$ at $8 \mathrm{~h}$ after scratching (t8) and was completed at $24 \mathrm{~h}$. In contrast, K5-GR MPKs exhibited $33.5 \%$ migration at $\mathrm{t} 8$, a marked delay that was still very prononunced at $\mathrm{t} 24$ $(44.2 \%)$. For comparative purposes, we evaluated the response of wt MPKs to the GC-analogue Dex and EGF (Fig. 4). As previously described (Lee et al., 2005), Dex delayed and EGF accelerated wt keratinocyte migration at 18 (35.2\% and $100 \%$, respectively). At t24, retarded migration elicited by Dex treatment was approximately $83.6 \%$. Note that constitutive GR overexpression in K5-GR MPKs elicited effects similar to Dex treatment in wt cells. The retarded migration of K5-GR MPKs was only slightly potentiated by Dex treatment (data not shown). K5-GR keratinocytes also responded to EGF although with delayed kinetics as compared to wt cells $(64.7 \%$ and $97.3 \%$ at t8 and $t 24$, respectively).

\section{Discussion}

During mammalian embryogenesis, the surface ectoderm gives rise to the corneal and conjunctival epithelia and the epidermis of the eyelid. Eyelid formation takes place during mouse embryonic days $E 15.5$ to $E 16.5$ and requires the proliferation and migration of epithelial cells to cover the ocular surface, acting as a protective barrier for normal eye development (Xia and Karin, 2004). This process is similar to epidermal formation which results in the acquisition of a competent barrier necessary to protect the organism from environmental damage (Segre, 2006). Our recent work has shown that GR is required for epidermal formation since its absence results in an immature epidermal barrier (Bayo et al., 2008). Analogously, histological evaluation of $\mathrm{GR}^{-/-}$embryos showed delayed and impaired eyelid closure, which took around $72-96 \mathrm{~h}$ to complete instead of $24 \mathrm{~h}$, as occurs in wt mice (Fig. 1). Delayed progression in total eyelid formation was around $50 \%$ in $\mathrm{GR}^{-/-}$E15.5 embryos and approximately $78.3 \%$ in E18.5 individuals relative to their wt counterparts (Fig. 2 and data not shown). The fact that GR modulates proliferation and apoptosis of eyelid epithelial cells (Fig. 2 A,B) adds to our previous findings in epidermis showing that this nuclear receptor regulates these processes in a cell-autonomous manner (Bayo et al., 2008) and highlights a general role of GR in epithelial morphogenesis. Our data also illustrate that these processes need to be temporally coordinated in order to complete proper eyelid formation. We found increased expression of markers of keratinocyte proliferation (K5, p63) in the eyelid epithelial cells of $\mathrm{GR}^{-/-}$embryos relative to wt, at a time where these markers should be restricted to the basal cell layer and a few suprabasal cells at the site of fusion (Fig. 1). We also observed incomplete differentiation of epithelial cells in $\mathrm{GR}^{-/}$embryos (Fig. 1, K10 and loricrin), most likely due to the absence of eyelid fusion, which should precede terminal differentiation (Zhang et al., 2005).

Our previous studies reported an EOB phenotype in K5-GR 
and K5-GR-TR embryos that was indeed the most consistent epithelial defect found in both transgenic mouse models (Cascallana et al., 2003; Donet et al., 2008). In addition, our data demonstrated that the transrepression function of the GR is sufficient to cause these epithelial anomalies (Donet et al., 2008). It was indeed surprising to find open eyelids in GR gain- and lossof-function mouse models although, undoubtedly, it further supports a key role for this transcription factor in ocular epithelial morphogenesis. Since $\mathrm{GR}^{-/-}$embryos exhibit increased corticosterone levels due to a feedback mechanism mediated by the hypothalamus-pituitary-adrenal axis (Cole et al., 1995 and our unpublished data), it is possible that elevated hormone levels may be acting through the mineralocorticoid receptor (MR) in $\mathrm{GR}^{-/-}$ ocular epithelial cells and thus mimick increased GC-signaling, even in the absence of GR. Supporting this hypothesis, mice overexpressing the MR in keratinocytes (K5-MR) showed an EOB phenotype virtually identical to that of K5-GR and K5-GR-TR embryos (Sainte Marie et al., 2007). The similarities between K5GR and K5-MR mouse models extend to epidermal defects, including abnormal hair follicle formation and hypoplastic epidermis (Sainte Marie et al., 2007). GR and MR recognize the same hexameric DNA response elements and, theoretically, could transactivate the same gene promoters (Kumar and Litwack, 2009). A subset of epithelial-specific genes may be regulated in common by both nuclear receptors. In this regard, our data show that GR represses egfr transcription in skin (Fig. 3B). However, $\mathrm{MR}$ has been reported to upregulate egfr mRNA expression in other cell types (Grossman et al., 2007).

GR exerts many of its biological actions through interactions with distinct signaling pathways, including MAPK/AP-1 and NFkappaB (reviewed in De Bosscher and Haegeman, 2009). Two main pathways have been involved in eyelid formation, the MEKK1/JNK/C-Jun pathway which transductes TGF- $\beta$ and activin signals and the TGF- $\alpha / E G F R / E R K$ pathway; both result in AP-1 activation (revised in Xia and Karin, 2004). EGFR plays a key role in eye morphogenesis and, accordingly, disruption of the EGFR locus resulted in an EOB phenotype as well as immature development of skin epithelial cells, teeth, lung and gastrointestinal tract (reviewed in Sibilia et al., 2007). We analyzed whether EGFR was abnormally expressed and/or activated in $\mathrm{GR}^{-/-}$embryos, and also investigated alterations in its downstream effector ERK. The expression pattern of both phosphorylated EGFR and ERK demonstrated sustained activation in $\mathrm{GR}^{-/-}$E18.5 embryos, a timepoint at which these proteins should be normally restricted to the more differentiated epithelial cells at the fused eyelids (Fig. 3). Sustained expression of ERK and $\mathrm{K} 6$ in the eyelids of $\mathrm{GR}^{-/-} \mathrm{E} 18.5$ mice is consistent with previous reports in vitro showing that EGFsignaling can induce the expression of K6 through AP-1 sites in its promoter (Lee et al., 2005).

The process of eyelid closure parallels skin wound healing, which also requires proliferation and migration to complete reepithelization across the wound (reviewed in Martin and Parkhurst, 2004; Barrientos et al., 2008). The expression of the migrationassociated keratin $\mathrm{K} 6$ increases during wound healing in adult skin and is required for normal re-epithelialization (Wong and Coulombe, 2003; Wojcik et al., 2000). Activated GR can inhibit the expression of specific keratins through several mechanisms involving transcriptional and non-transcriptional events (Radoja et al., 2000). GCs were shown to cause cytoskeleton remodeling by repressing K6/K16 expression and thus, inhibiting keratinocyte migration and causing deregulated growth and differentiation (Stojadinovic et al., 2005). These GR actions are relevant during the wound healing process, whereby $\mathrm{K} 6$ expression in suprabasal keratinocytes at the wound's edge is repressed once the epidermis covers the wound. This occurs through antagonism between GR and EGFR and involves activation of $\beta$-catenin and c-myc and blockade of EGF effects through the formation of a complex consisting in four GR monomers, $\beta$-catenin and coactivatorassociated-arginine-methyltransferase-1 (Lee et al., 2005; Stojadinovic et al., 2005).

So far, the in vivo cross-talk between GR/EGFR in eyelid formation had not been previously investigated. Our data in vivo show that $\mathrm{GR}$ is a master regulator required for the spatiotemporal control of the EGFR/MAPK/AP-1 signaling at the leading edge of eyelid keratinocytes and suggest that this interference is required for normal eyelid development.

\section{Materials and Methods}

\section{Animals}

All mice were handled in accordance with the current Spanish and European normative which governs research with animals (Real Decreto 1201/2005, B.O.E. \#252, 10 of October, 2005 and Convenio Europeo 12-3 del 18/3/1986).

$\mathrm{GR}^{-/-}$and K5-GR mice have been previously reported (Cole et al., 1995; Pérez et al., 2001). GR ${ }^{+/-}$hemizygous mice (B6D2/F1) intercrosses were programmed to obtain $\mathrm{GR}^{-/}$, $\mathrm{GR}^{+/-}$and $\mathrm{GR}^{+/+}$mice. Embryos were obtained by cesarean derivation at the indicated day post-conception (dpc; the morning of the day that the vaginal plug was seen was considered as day $0.5 \mathrm{pc}$.). For histopathological evaluation, we analyzed embryos of different timepoints from $\mathrm{GR}^{-/}, \mathrm{GR}^{+/-}$and $\mathrm{GR}^{+/+}$genotypes $(n=43)$. For preparation of mouse primary keratinocytes (MPKs) and wound scratch assays, skin from K5-GR mice and wt newborn littermates was excised and processed $(n=38)$.

\section{Antibodies}

The antibodies used included rabbit polyclonal antibodies against keratin K5 (PRB-160P), K6 (PRB-169P), K10 (PRB-159P) and loricrin (PRB-145P) from Covance (Babco, Berkeley, CA). Antibodies against p63 (sc-404), p-c-jun (sc-822) and EGFR (sc-03) were from Santa Cruz Biotechnology, Inc., (Santa Cruz, CA) and anti-p-EGFR (53A5), anti-pERK (Thr202/Tyr204) (\#4376) and p-JNK (Thr183/Tyr185) (\# 9251) were purchased from Cell Signaling (Cell Signaling Technology Inc., Beverley, MA). Secondary biotin-conjugated anti-rabbit or anti-mouse antibodies were from Jackson ImmunoResearch (Jackson ImmunoResearch Laboratories, Inc. West Grove, PA).

\section{Histological and Immunohistochemical analysis}

Embryo heads were fixed in $4 \%$ paraformaldehyde (PFA) or $70 \%$ ethanol and embedded in paraffin. Consecutive 3 to $4 \mu \mathrm{m}$-thick sections were obtained. For histopathology, sections were stained with hematoxylin/eosin. Prior to immunostaining, paraffin sections were dewaxed and microwaved in $10 \mathrm{mM}$ citrate solution. For immunohistochemistry, paraffin sections were blocked with $5 \%$ fetal bovine serum, and then incubated with the primary antibody for at least one hour. Slides were washed three times with PBS, and then incubated with conjugated secondary antibodies for $1 \mathrm{~h}$. Finally, the reaction was visualized with the Avidin-BiotinComplex (ABC) kit from DAKO (Vectastain Elite, Vector Laboratories, Inc, Burlingame, CA) using diamino-benzidine as chromogenic substrate for peroxidase. Slides were mounted and analyzed by light microscopy (Leica DM RXA2), and microphotographs were taken at the indicated magnification. 


\section{In vivo epithelial BrdU labeling}

Epithelial cell proliferation was measured by i.p. injection of BrdU (130 $\mu \mathrm{g} / \mathrm{g}$ of body weight, Roche) into pregnant female mice $1 \mathrm{~h}$ before sacrifice. BrdU incorporation was detected by immunohistochemistry of paraffin-embedded sections using a mouse anti-BrdU monoclonal antibody (biotest, Roche) followed by hematoxylin counterstaining. The number of BrdU-positive cells and the number of total cells was determined per $200 \mu \mathrm{m}$ of interfollicular epithelium in each section. Experiments were performed at least in five individuals of each genotype and differences were assessed by using the t test, with statistical significance when $p<0.05$

\section{Analysis of apoptosis in tissue sections}

To detect individual apoptotic cells in paraffin-embedded tissue sections, the In situ Cell Death Detection kit (Roche) was used, following manufacturer's recommendation. Paraffin sections immersed in $0.1 \mathrm{M}$ citrate buffer, $\mathrm{pH} 6$ were microwave-irradiated for $5 \mathrm{~min}$, and then rinsed with PBS prior to the TUNEL reaction. Four $15.5 \mathrm{dpc}$ embryos of each genotype were examined.

\section{Measurements of eyelid formation, leading edge formation and root formation}

We have quantitated the differences in eyelid formation of $\mathrm{GR}^{-/-} v s \mathrm{wt}$ E15.5 littermates, following the methodology described by Mine et al. These measurements were done in slides that were stained with $\mathrm{K} 6$, in order to delimitate the migrating epitheliall cells as K6-positive cells. This migration distance was considered as parameter $a$; the distance between the axis delimitating the conjunctival sac and the first K6-stained cells was denominated as $b$; the distance between the axis delimitating the conjunctival sac and the center of the cornea was denominated as $c$. The percentage of root formation was calculated as [b/c] $\times 100$; percentage of leading edge formation was calculated as $[\mathrm{a} / \mathrm{c}] \times 100$, and the percentage of eyelid formation was $[(a+b) / c] \times 100$. Calculations were done in five individuals of each genotype and differences considered to be statistically significant when $p<0.05$.

\section{MPK isolation, culture and wound scratch}

MPK isolation was performed as previously described (Bayo et al., 2008). Briefly, skin was peeled off, incubated in $0.25 \%$ trypsin to separate the epidermis from the dermis and homogenized. MPKs were pooled (using at least two mice per point) and $10^{6}$ cells were plated into one 35 $\mathrm{mm}$ diameter collagen coated petri dish (BD Biosciences) and cultured at $37^{\circ} \mathrm{C}$ in standard medium. After $24 \mathrm{~h}$, the medium was replaced with complete low calcium medium and cells were grown until confluency. The composition of standard medium was: Essential modified Eagle's medium EMEM (BioWhitakker, Inc., Walkersville, MD), supplemented with $4 \%$ fetal calf serum (FCS, BioWhitakker, Inc.) plus $0.6 \mathrm{mM} \mathrm{CaCl}_{2}$ and antibiotics. To prepare low-calcium medium, FCS was depleted of divalent cations by treatment with Chelex deionizing resin (BioRad, Hempstead, UK) and supplemented with $\mathrm{CaCl}_{2}$ to a final concentration of approximately $0.05 \mathrm{mM}$. EGF (Sigma, St. Louis, MO) $(10 \mathrm{ng} / \mathrm{ml})$ and antibiotics were added to growth medium.

For wound scratch assays, MPKs were incubated in EMEM/1\% FBS $\mathrm{O} / \mathrm{N}$, and then treated with mitomycin $(10 \mu \mathrm{g} / \mathrm{ml})$ for $1 \mathrm{~h}$. Next, cells were wounded with a yellow tip, treated as indicated and cell migration followed up for 8-24h. Experiments were performed in triplicate and mean value \pm SD estimated. Vehicle, Dexamethasone (Dex, Sigma, St. Louis, MO, 100 $\mathrm{nM})$ or EGF $(25 \mathrm{ng} / \mathrm{ml})$ were added for the indicated times to confluent wt MPKs.

For each wound scratch experiment, the surface area that remained uncovered by the cells for each time-point and condition was quantitated (Adobe Photoshop 8.1.0). These measurements were expressed as a percentage of distance coverage by cells moving into the scratch wound area $8 \mathrm{~h}$ and $24 \mathrm{~h}$ after wounding. Six images were analyzed per condition and time-point; then, averages and standard deviations were calculated.

\section{RNA preparation and quantitative $R T-P C R$}

Total RNA was isolated from back skin of $\mathrm{GR}^{-/-}$and control littermates (four animals of each genotype) by using Trizol reagent (Invitrogen, Molecular Probes, Eugene, Oregon), following manufacturer's recommendations. Reverse transcription was performed by using $1 \mu \mathrm{g}$ of RNA and oligo-dT (Fermentas Inc., Burlington, Canada) followed by qPCR using specific oligonucleotides for egfr

Forward, 5'-CAAAGTGATGTCTGGAGCTAT-3';

Reverse, 5'CTTGCTGGGATTCCATCATAAG-3،. Technical triplicates were performed and mean value \pm SD estimated.

\section{Acknowledgements}

This work was supported by grant SAF2008-00540 of the Ministerio de Ciencia e Innovación from the Spanish government and Fundación Ramón Areces (050507070007). LMS is recipiente of a contract JAE-Doc co-funded by European Funds. We are grateful to Prof. Günther Schütz for providing us with $\mathrm{GR}^{-/}$mice and ackowledge Fátima Riveiro for histological work.

\section{References}

ADAMS, M., MEIJER, O.C., WANG, J., BHARGAVA, A. and PEARCE, D. (2003) Homodimerization of the glucocorticoid receptor is not essential for response element binding: activation of the phenylethanolamine $N$-methyltransferase gene by dimerization-defective mutants. Mol Endocrinol 17: 2583-2592.

BARRIENTOS, S., STOJADINOVIC, O., GOLINKO, M.S., BREM, H. and TOMICCANIC, M. (2008). Growth factors and cytokines in wound healing. Wound Repair Regen 16: 585-601.

BAYO, P., SANCHIS, A., BRAVO, A., CASCALLANA, J.L., BUDER, K., TUCKERMANN, J., SCHÜTZ, G. and PÉREZ, P. (2008). Glucocorticoid receptor is required for skin barrier competence. Endocrinology 149: 1377-1388.

CASCAllanA, J.L., BRAVO, A., PAGE, A., BUDUNOVA, I., SLAGA, T.J., JORCANO, J.L., and PÉREZ, P. (2003). Disruption of eyelid and cornea development by targeted overexpression of the glucocorticoid receptor. Int $J$ Dev Biol 47: 59-64.

CASCALLANA, J.L., BRAVO, A., DONET, E., LEIS, H., JORCANO, J.L., and PÉREZ, P. (2005) Ectoderm-targeted overexpression of the glucocorticoid receptor induces hypohidrotic ectodermal dysplasia Endocrinology 146: 26292638.

COLE, T.J, BLENDY, A.P., MONAGHAN, K., SCHMID, W., AGUZZI, A., FANTUZZI, G., HUMMLER, E., UNSICKER, K. and SCHÜTZ, G. (1995). Targeted disruption of the glucocorticoid receptor gene blocks adrenergic chromaffin cell development and severely retards lung maturation. Genes Dev 9: 1608-1621.

DE BOSSCHER, K. and HAEGEMAN, G. (2009). Minireview: latest perspectives on antiinflammatory actions of glucocorticoids. Mol Endocrinol 23: 281-289.

DONET, E., BOSCH, P., SANCHIS, A., BAYO, P., RAMÍREZ, A., CASCALLANA, J.L., BRAVO, A. and PÉREZ, P. (2008). Transrepression function of the glucocorticoid receptor regulates eyelid development and keratinocyte proliferation but is not sufficient to prevent skin chronic inflammation Mol Endocrinology 22: 799- 812

GRossmanN, C., KRUG, A.W., FREUdingeR, R., MILDENBERGER, S., VOELKER, K. and GEKLE M. (2007). Aldosterone-induced EGFR expression: interaction between the human mineralocorticoid receptor and the human EGFR promoter. Am J Physiol Endocrinol Metab 292: 1790-1800.

KAUFMAN, M.H. and BARD, J.B.L. (1999). The anatomical basis of mouse development. San Diego, Academic Press.

KUMAR, R. and LITWACK, G. (2009). Structural and functional relationships of the steroid hormone receptors' N-terminal transactivation domain. Steroids 74 : 877-883.

LEE, B., VOUTHOUNIS, C., STOJADINOVIC, O., BREM, H., IM, M. AND TOMICCANIC, M. (2005). From an Enhanceosome to a Repressosome: Molecular Antagonism between Glucocorticoids and EGF Leads to inhibition of Wound Healing. J Mol Biol 345: 1083-1097.

MARTIN, P. and PARKHURST, S.M. (2004). Parallels between tissue repair and embryo morphogenesis. Development 131: 3021-3034. 
MINE, N., IWAMOTO, R. and MEKADA, E. (2005). HB-EGF promotes epithelial cell migration in eyelid development. Development 132: 4317-4326.

PEREZ, P., PAGE, A., BRAVO, A., DEL RÍO, M., GIMÉNEZ-CONTI, I., BUDUNOVA, I., SLAGA, T.J. and JORCANO, J.L. (2001). Altered skin development and impaired proliferative and inflammatory responses in transgenic mice overexpressing the glucocorticoid receptor. FASEB J 15: 2030-2032.

RADOJA, N., KOMINE, M., JHO, S.H., BLUMENBERG, M. and TOMIC-CANIC, M. (2000). Novel mechanism of steroid action in skin through glucocorticoid receptor monomers. Mol Cell Biol 20: 4328-4339.

REICHARDT, H.M., KAESTNER, K.H., TUCKERMANN, J., KRETZ, O., WESSELY, O., BOCK, R., GASS, P., SCHMID, W., HERRLICH, P., ANGEL, P. and SCHÜTZ, G. (1998). DNA binding of the glucocorticoid receptor is not essential for survival. Cell 93: 531-541.

REVOLLO, J.R. and CIDLOWSKI, J.A. (2009). Mechanisms Generating Diversity in Glucocorticoid Receptor Signaling. Ann NY Acad Sci 1179: 167-178.

ROGATSKY, I., WANG, J.C., DERYNCK, M.K., NONAKA, D.F., KHODABAKHSH, D.B., HAGG, C.M., DARIMONT, B.D., GARABEDIAN, M.J. and YAMAMOTO, K.R. (2003). Target-specific utilization of transcriptional regulatory surfaces by the glucocorticoid receptor. Proc Natl Acad Sci USA 100: 13845-13850.

SAINTE MARIE, Y., TOULON, A., PAUS, R., MAUBEC, E., CHERFA, A., GROSSIN, M., DESCAMPS, V., CLEMESSY, M., GASC, J.M., PEUCHMAUR, M., GLICK, A., FARMAN, N. and JAISSER, F. (2007). Targeted skin overexpression of the mineralocorticoid receptor in mice causes epidermal atrophy, premature skin barrier formation, eye abnormalities, and alopecia. Am J Pathol 171: 846-860.

SEGRE, J.A. (2006). Epidermal barrier formation and recovery in skin disorders. $J$ Clin Invest 116: 1150-1158.
SIBILIA, M., KROISMAYR, R., LICHTENBERGER, B.M., NATARAJAN, A., HECKING, M. and HOLCMANN, M. (2007). The epidermal growth factor receptor: from development to tumorigenesis. Differentiation 75: 770-787.

STOJADINOVIC, O., BREM, H., VOUTHOUNIS, C., LEE, B., FALLON, J., STALLCUP, M., MERCHANT, A., GALIANO, R.D. and TOMIC-CANIC, M. (2005). Molecular Pathogenesis of Chronic Wounds. The Role of b-Catenin and c-myc in the Inhibition of Epithelialization and Wound Healing. Am J Pathol 167: 59-69.

WINTERMANTEL, T.M., BERGER, S., GREINER, E.F. and SCHÜTZ, G. (2004). Genetic dissection of corticosteroid receptor function in mice. Horm Metab Res 36: 387-391.

WOJCIK, S.M., BUNDMAN, D.S. and ROOP, D.R. (2000). Delayed Wound Healing in Keratin 6a Knockout Mice. Mol Cell Biol 20: 5248-5255.

WONG, P. and COULOMBE, P.A. (2003). Loss of keratin 6 (K6) proteins reveals a function for intermediate filaments during wound repair. J Cell Biol 163: 327337.

XIA, Y. and KARIN, M. (2004). The control of cell motility and epithelial morphogenesis by Jun kinases. Trends Cell Biol 14: 94-101.

ZENZ, R., SCHEUCH, H., MARTIN, P., FRANK, C., EFERL, R., KENNER, L., SIBILIA, M. and WAGNER, E.F. (2003). C-Jun Regulates Eyelid Closure and Skin Tumor Development through EGFR Signaling. Dev Cell 4: 879-889.

ZHANG, H., HARA, M., SEKI, K., FUKUDA, K and NISHIDA, T. (2005). Eyelid Fusion and Epithelial Differentiation at the Ocular Surface During Mouse Embryonic Development. Jpn J Ophthalmol 49: 195-204. 


\section{Further Related Reading, published previously in the Int. J. Dev. Biol.}

See our recent Special Issue Placenta edited by Joan S. Hunt and Kent L. Thornburg at: http://www.ijdb.ehu.es/web/contents.php?vol=54\&issue=2-3

Corneal development associated with eyelid opening

James D. Zieske

Int. J. Dev. Biol. (2004) 48: 903-911

Analysis of mouse eye development with chimeras and mosaics J. Martin Collinson, Robert E. Hill and John D. West

Int. J. Dev. Biol. (2004) 48: 793-804

Disruption of eyelid and cornea development by targeted overexpression of the glucocorticoid receptor

José Luis Cascallana, Ana Bravo, Angustias Page, Irina Budunova, Thomas J Slaga, José L Jorcano and Paloma Pérez

Int. J. Dev. Biol. (2003) 47: 59-64

\section{PPAR expression and function during vertebrate development}

Liliane Michalik, Béatrice Desvergne, Christine Dreyer, Mathilde Gavillet, Ricardo N Laurini and Walter Wahli

Int. J. Dev. Biol. (2002) 46: 105-114

Role of the retinoic acid receptor beta (RARbeta) during mouse development N B Ghyselinck, V Dupé, A Dierich, N Messaddeq, J M Garnier, C Rochette-Egly, P Chambon and M Mark

Int. J. Dev. Biol. (1997) 41: 425-447

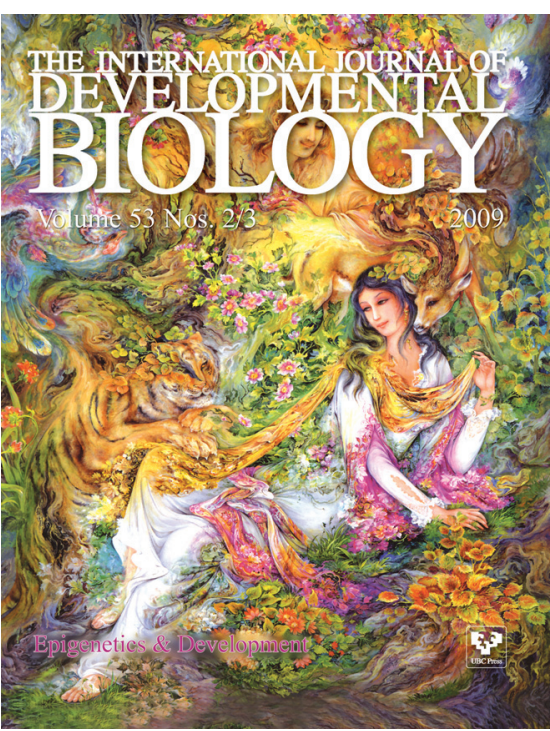

5 yr ISI Impact Factor $(2008)=3.271$
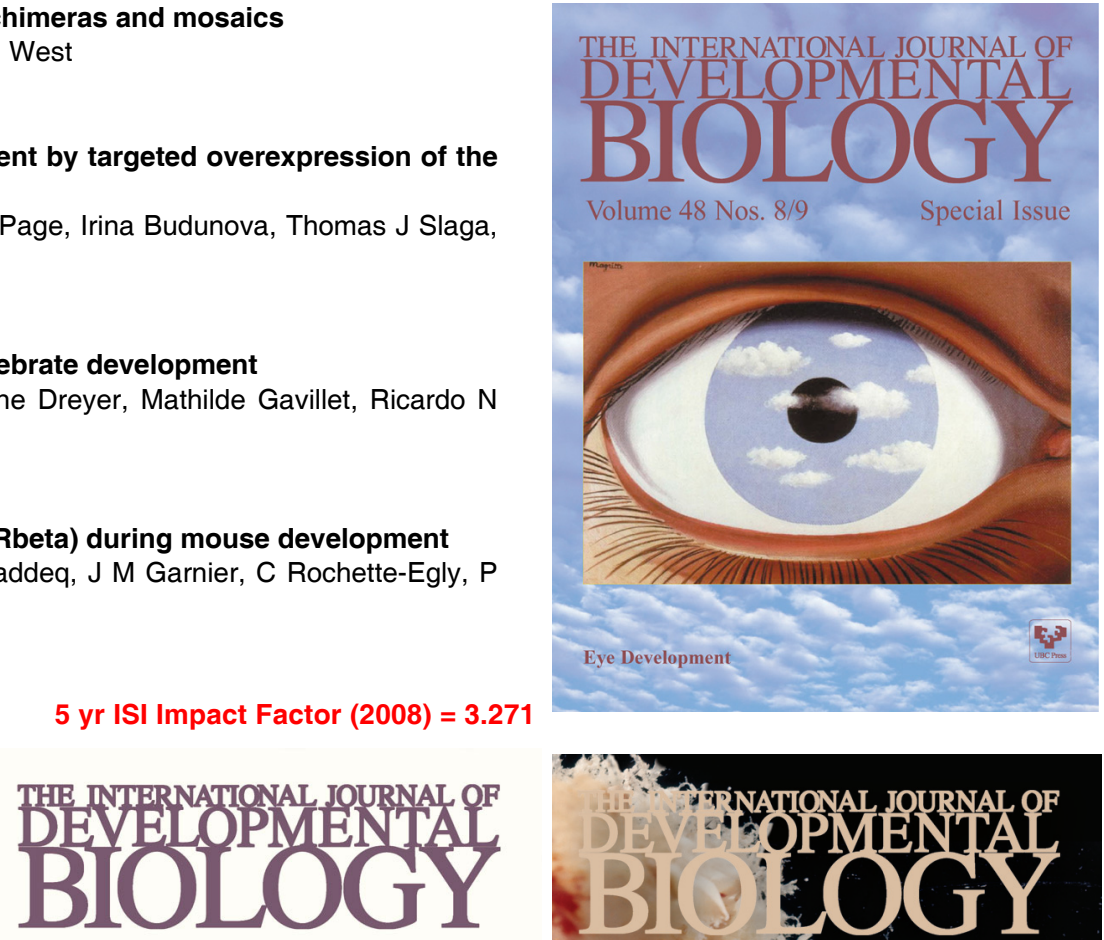

Volume 54 Nos. $6 / 7$

Special Issue
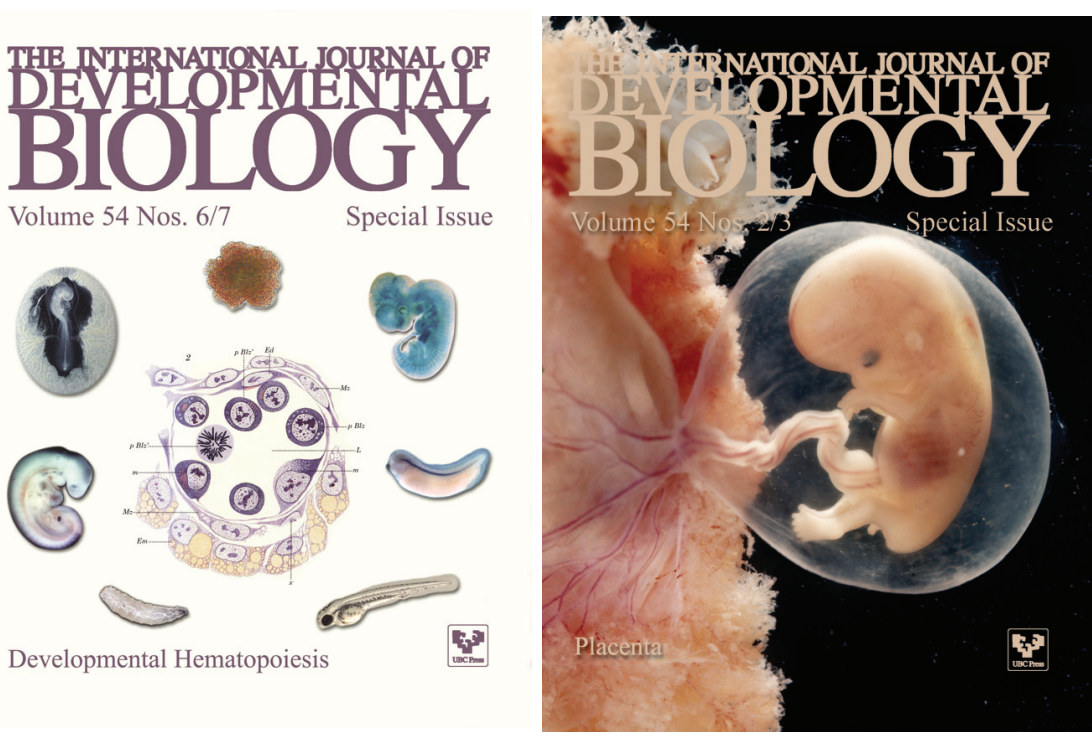